\title{
Algorithm for Obtaining Optimal Path in Three-objective Network with a Reduction of Search Space
}

\author{
Natsumi TAKahashi ${ }^{1}$, Hisashi Yамамото ${ }^{1}$, Tomoaki AkIBA ${ }^{2}$, and Xiao XIAO ${ }^{1}$ \\ ${ }^{1}$ Faculty of System Design, Tokyo Metropolitan University, 6-6 Asahigaoka, Hino, Tokyo 191-0065, Japan \\ ${ }^{2}$ Faculty of Social Systems Science, Chiba Institute of Technology, 2-17-1 Tsudanuma, Narashino, Chiba 275-0016, Japan
}

\begin{abstract}
Many network systems have been applied extensively in the real world, for example, a route guiding system and an optimal path of a network connection to Internet services. Many papers have dealt with networks with two specific nodes. Each edge has criteria, for example distances, costs, and the time required. The optimal path problem aims to obtain the particular path that has a minimum value of criteria. In this paper, we consider the optimal path problem for a three-objective network. In a multi-objective network, edges have two or more criteria. Few cases exist where multi-criteria are optimized at the same time. Thus, it is worth obtaining Pareto solutions as optimal paths in multi-objective networks. An extended Dijkstra's algorithm was proposed to obtain Pareto solutions of two- and threeobjective networks. Even if we use this existing algorithm, obtaining optimal paths is difficult in large networks that have many nodes. Therefore, more efficient algorithms than the extended Dijkstra's algorithm were proposed. However, these algorithms cannot obtain all Pareto solutions. Thus, an efficient and exact algorithm that can obtain all Pareto solutions is required. We describe an essential and effective idea in reducing search space. Using this idea, our algorithms can set bounds to search within less space than the extended Dijkstra's algorithm and obtain all Pareto solutions. For evaluating the algorithms, we conducted numerical experiments. As a result, we show our algorithms are more efficient than the extended Dijkstra's algorithm.
\end{abstract}

Key Words : Multi-objective Network, Extended Dijkstra's Algorithm, Optimal Path Problem, Pareto Solutions.

\section{Introduction}

Many network systems have been applied extensively in the real world, for example, route guiding systems, scheduling of a production and distribution management system, and search systems for optimal routes on Internet services. Many papers have dealt with networks with two specific nodes. When a network has two specific nodes (a start node and terminal node), many paths from the start node to the terminal node can be generated by allocation of edges. Each edge has criteria, for example, distances, costs, and the time required. The optimal path problem aims to obtain the particular path that has the minimum value of criteria. We call such a path the optimal path.

Dijkstra's and the Bellman-Ford algorithms[1][2][3] are well known to be effective in solving the optimal path problem for single-objective networks. These algorithms are used to find the shortest path between two specific nodes in a network. However, these algorithms can be applied to networks with only one criterion.

In this paper, we consider multi-objective networks, in which each edge has two and more criteria. Several studies have been conducted on multi-objective networks; one criterion is considered to be an objective function, and another is considered to be the constraints. Hara et al.[4] proposed a route planning method in car navigation systems that uses a multi-objective genetic

Corresponding Author: Natsumi Takahashi

Faculty of System Design, Tokyo Metropolitan University, Tokyo, 1900065 Japan

takahashi-natsumi@ed.tmu.ac.jp

(Received March 27, 2015)

(Revised May 21, 2015)

(Accepted May 28, 2015) algorithm (MOGA) for multi-objective networks with subjective comfort. Also, Kambayashi et al.[5] proposed a MOGA for selecting a shorter route with a lower number of intersections. The "route" in their studies is the same as the "path" in our study. Aneja et al.[6] proposed an extended Dijkstra's algorithm for an optimal path problem with a constraint condition, and Miyamoto[7] proposed a more efficient algorithm than Aneja et al.

However, a few studies have been conducted on obtaining Pareto solutions in multi-objective networks. There are few cases where all criteria, which the edge has, are optimal (minimized) at the same time because each criterion conflicts with others. Therefore, obtaining Pareto solutions as optimal paths in multi-objective networks is worthwhile. Akiba et al.[8] proposed an extended Dijkstra's algorithm that obtains all Pareto solutions in two-objective networks by applying the algorithm for an optimal path with a constraint condition[7]. Takahashi et al.[9] proposed an efficient algorithm for all Pareto solutions in two-objective networks with a reduction in the search space. Akiba et al.[10] also applied an extended Dijkstra's algorithm by Miyamoto[7] to a three-objective network.

In this paper, we consider a three-objective network. When networks have multi-criteria, the search for paths uses a large memory area and takes much computing time in the extended Dijkstra's algorithm. Even if we use this algorithm, obtaining optimal paths is difficult in large networks that have many nodes. Akiba et al.[10] proposed more efficient algorithms than the extended Dijkstra's algorithm. However, their efficient algorithms cannot obtain all Pareto solutions. Thus, an efficient and exact algorithm that can obtain all Pareto solutions is re- 
quired. For such a three-objective network, we propose algorithms for all Pareto solutions, based on the extended Dijkstra's algorithm[10] and the idea of Takahashi et al.[9]. We propose the essential and effective idea of reducing search space. By using this idea, our algorithms can set the bounds to search within less space than the extended Dijkstra's algorithm and can obtain all Pareto solutions.

This paper is organized as follows. In section 2, our considered problem is thoroughly described. In section 3, the extended Dijkstra's algorithm is explained because our algorithms are obtained by improving this algorithm. In section 4 , our proposed algorithms are described in detail. In section 5, we explain the results of numerical experiments to evaluate our algorithms. As a result, we show our algorithms are more efficient than the extended Dijkstra's algorithm.

\section{Definition of Problem}

\subsection{Network system}

This section defines some notations to state our problem, as follows. $V$ denotes a set of nodes and $E$ a set of directed edges $E=\left\{e_{1}, \cdots \cdot e_{n}\right\}$, where $n$ is the number of edges, and $e_{j}$ is the $j$-th edge for $j=1,2, \cdots, n$. Let $G=(V, E)$ be a given network. Node $s(\in V)$ indicates the start node, and $t(\in V)$ indicates the terminal node in a network. Now, we consider a target goes through edges from node $s$ to node $t$. We call the set of such edges a path from node $s$ to node $t$. Edge $e(\in E)$ has a cost vector $\left(c_{1 e}, c_{2 e}, c_{3 e}\right)$, where element $c_{i e}$ is the cost related to the $i$-th objective function of edge $e$ for $i=1,2,3$ and where all $c_{i e}$ take nonnegative values. Also, we suppose $\operatorname{costs} c_{1 e}, c_{2 e}$, and $c_{3 e}$ occur if the target goes through edge $e$. For example, if a target goes through the path that consists of edges $e_{1}$ and $e_{2}$, costs $c_{1 e_{1}}+c_{1 e_{2}}, c_{2 e_{1}}+c_{2 e_{2}}$, and $c_{3 e_{1}}+c_{3 e_{2}}$ occur.

\subsection{Optimal path search problem of three-objective net- work}

For $j=1,2, \cdots, n$, let $x_{j} \in\{0,1\}$ be a binary variable, where $x_{j}=1$ if the target goes through edge $e_{j}$, and $x_{j}=0$ if not. By using these variables, we define $n$-dimensional vector $\mathbf{x}=$ $\left(x_{1}, x_{2}, \cdots, x_{n}\right)$.

A target can go through only one edge at the same time. Therefore, the following equations hold. For $v \in V$,

$$
\sum_{\left\{e_{j} \in E \mid \operatorname{start}\left(e_{j}\right)=v\right\}} x_{j}-\sum_{\left\{e_{j} \in E \mid \operatorname{end}\left(e_{j}\right)=v\right\}} x_{j}=\left\{\begin{array}{cc}
1, & v=s, \\
-1, & v=t, \\
0, & \text { otherwise },
\end{array}\right.
$$

where $\operatorname{start}(e)$ and $\operatorname{end}(e)$ mean the start and end node of an edge $e$, respectively.

Note that the $n$-dimensional vector $\mathbf{x}$, whose elements satisfy equation (1), can express any paths from start node $s$ to terminal node $t$. Then, $X$ denotes a set of paths from start node $s$ to terminal node $t$, that is,

$X \equiv\{\mathbf{x} \mid$ elements of $\mathbf{x}$ satisfy equation (1) $\}$.

Next, we define the following function. For $i=1,2,3$, $g_{i}(\mathbf{x})=\sum_{e_{j} \in E} c_{i j} x_{j}$.

$g_{i}(\mathbf{x})$ means $i$-th total cost, when the target goes through a path $\mathbf{x}$.

Next, we describe the definition of Pareto solutions considered in this study.

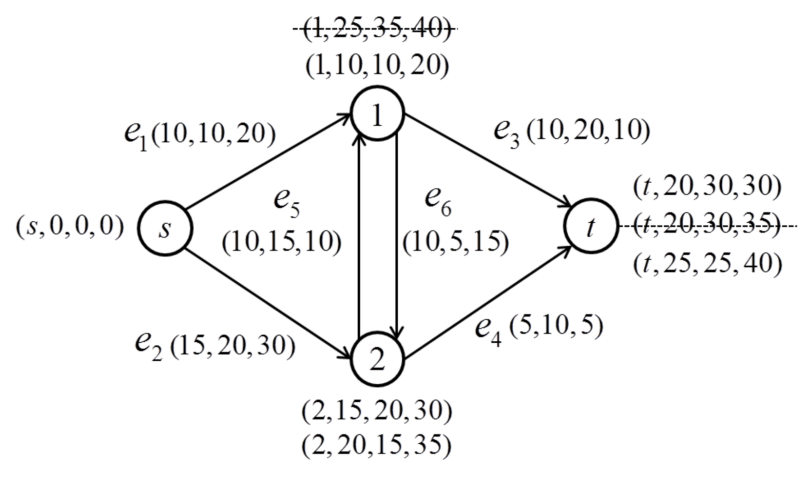

Fig. 1 Procedure for label calculation

\section{Definition of the Pareto solutions}

We consider two paths, $\mathbf{x}, \mathbf{x}^{\prime} \in X$. We say $\mathbf{x}$ dominates $\mathbf{x}^{\prime}$ when $g_{i}(\mathbf{x}) \leq g_{i}\left(\mathbf{x}^{\prime}\right)$ for $i=1,2,3$ and when at least one strict inequality holds. Then, $\mathbf{x}$ is defined as the Pareto solution when no path dominates $\mathbf{x}$.

Therefore, a three-objective optimal path problem in this study can be expressed as follows.

\section{Definition of optimal path problem}

$$
\begin{aligned}
& g_{1}(\mathbf{x})=\sum_{e_{j} \in E} c_{1 j} x_{j} \rightarrow \min , \\
& g_{2}(\mathbf{x})=\sum_{e_{j} \in E} c_{2 j} x_{j} \rightarrow \min , \\
& g_{3}(\mathbf{x})=\sum_{e_{j} \in E} c_{3 j} x_{j} \rightarrow \min , \\
& \text { s.t. } \\
& \quad \sum_{\left\{e_{j} \in E \mid \text { start }\left(e_{j}\right)=v\right\}} x_{j}-\sum_{\left\{e_{j} \in E \mid \text { end }\left(e_{j}\right)=v\right\}} x_{j}=\left\{\begin{array}{cc}
1, & v=s, \\
-1, & v=t, \\
0, & \text { otherwise, }
\end{array}\right.
\end{aligned}
$$

for $v \in V$.

\section{Extended Dijkstra's Algorithm}

The extended Dijkstra's algorithms for a constrained optimal path problem were proposed by Aneja et al.[6] and Miyamoto[7]. As described in section 1, Akiba et al.[8][10] proposed the extended Dijkstra's algorithm, which obtained all Pareto solutions in a two or three objective network by applying Miyamoto's algorithm. In this study, we improve the extended Dijkstra's algorithm by Akiba et al.[10] to obtain all Pareto solutions in the three-objective network. Before this, we explain the extended Dijkstra's algorithm in this section. First, the following notations are defined. For $v \in V$,

$\mathbf{x}_{v}: n$-dimensional vector, which indicates a path from start node $s$ to node $v$.

$W_{v}$ : set of adjacent nodes to node $v$.

And for $v \in V$ and $i=1,2,3$,

$l_{i v}$ : total cost related to the $i$-th objective function when a target goes through the path from node $s$ to node $v$, that is $l_{i v} \equiv$ $g_{i}\left(\mathbf{x}_{v}\right)$.

Next, we define the "label" $\left(v, l_{1 v}, l_{2 v}, l_{3 v}\right)$ as the combination of node $v$ and total costs $l_{1 v}, l_{2 v}$, and $l_{3 v}$. Let $L_{v}$ be a set of labels for node $v$. We consider two labels $\left(v, l_{1 v}, l_{2 v}, l_{3 v}\right)$ and $\left(v, l^{\prime}{ }_{1 v}, l^{\prime}{ }_{2 v}, l^{\prime}{ }_{3 v}\right)$. If for $i=1,2,3, l_{i v} \leq l^{\prime}{ }_{i v}$ and at least one strict inequality holds, then the label $\left(v, l_{1 v}, l_{2 v}, l_{3 v}\right)$ dominates the label $\left(v, l^{\prime}{ }_{1 v}, l^{\prime}{ }_{2 v}, l^{\prime}{ }_{3 v}\right)$. 
Figure 1 describes how to calculate labels. There are two paths from start node $s$ to node 1 . One is the path going through edge $e_{1}$. This path generates the label $(1,10,10,20)$ for node 1 . The other path uses edges $e_{2}$ and $e_{5}$. The label by this path is calculated as $(1,15+10,20+15,30+10)=(1,25,35,40)$. Compared with these two labels, $(1,10,10,20)$ dominates $(1,25,35,40)$. Therefore, $\{(1,10,10,20)\}$ is assigned to the set $L_{1}$. Similarly, $L_{2}=\{(2,15,20,30),(2,20,15,35)\}$. These labels of node 1 and node 2 generate the labels of terminal node $t$. In a similar way, $L_{t}=\{(t, 20,30,30),(t, 25,25,40)\}$. This is the procedure for calculating labels by using the extended Dijkstra's algorithm. The details of the extended Dijkstra's algorithm[10] are described as follows.

\section{Steps in extended Dijkstra's algorithm}

STEP 1: (Initialize) Set $L_{v} \leftarrow\{(s, 0,0,0)\}, W_{v} \leftarrow \phi, v \leftarrow s$. STEP 2: Obtain the set $W_{v}$ of adjacent nodes to node $v$. STEP 3: (Obtain the path to adjacent nodes)

STEP 3-1: Select node $\omega\left(\in W_{v}\right)$.

STEP 3-2: Select $\left(v, l_{1 v}, l_{2 v}, l_{3 v}\right) \in\left\{\left(\tau, l_{1 \tau}, l_{2 \tau}, l_{3 \tau}\right) \mid\right.$

$\left.\left(\left(\tau, l_{1 \tau}, l_{2 \tau}, l_{3 \tau}\right) \in L_{v}\right) \cap(\tau=v)\right\}$.

STEP 3-3: Calculate $\left(\omega, l_{1 v}+c_{1 e}, l_{2 v}+c_{2 e}, l_{3 v}+c_{3 e}\right)$

for edge e when start $(e)$ is $v$ and end $(e)$ is $\omega$, and set $\left(\omega, l_{1 \omega}^{*}, l_{2 \omega}^{*}, l_{3 \omega}^{*}\right) \leftarrow\left(\omega, l_{1 v}+c_{1 e}, l_{2 v}+c_{2 e}, l_{3 v}+c_{3 e}\right)$.

STEP 3-4: Compare $\left(\omega, l_{1 \omega}^{*}, l_{2 \omega}^{*}, l_{3 \omega}^{*}\right)$ with all elements of $L_{v}$. If label $\left(\omega, l_{1 \omega}^{*}, l_{2 \omega}^{*}, l_{3 \omega}^{*}\right)$ is the Pareto solution, $\left(\omega, l_{1 \omega}^{*}, l_{2 \omega}^{*}, l_{3 \omega}^{*}\right)$ is memorized to a set of $L_{v}$

STEP 3-5: Go to STEP 3-6 if all $\left(v, l_{1 v}, l_{2 v}, l_{3 v}\right)$ are selected in STEP 3-2. Go to STEP 3-2 otherwise.

STEP 3-6: Go to STEP 4 if all nodes in $W_{v}$ (that is, adjacent nodes to $v$ ) are selected in STEP 3-1. Go to STEP 3-1 otherwise.

STEP 4: (Obtain the path from the known path to adjacent nodes and calculate the labels)

STEP 4-1: Select node $\omega\left(\in W_{v}\right)$.

STEP 4-2: Select label $\left(\sigma, l_{1 \sigma}, l_{2 \sigma}, l_{3 \sigma}\right) \in L_{v}$ for node $\sigma$, which is one of all nodes connecting with node $\omega$.

STEP 4-3: Calculate $\left(\omega, l_{1 \sigma}+c_{1 e}, l_{2 \sigma}+c_{2 e}, l_{3 \sigma}+c_{3 e}\right)$ for edge $e$ when $\operatorname{start}(e)$ is $\sigma$ and end $(e)$ is $\omega$, and set $\left(\omega, l_{1 \omega}^{*}, l_{2 \omega}^{*}, l_{3 \omega}^{*}\right) \leftarrow\left(\omega, l_{1 \sigma}+c_{1 e}, l_{2 \sigma}+c_{2 e}, l_{3 \sigma}+c_{3 e}\right)$.

STEP 4-4: Compare $\left(\omega, l_{1 \omega}^{*}, l_{2 \omega}^{*}, l_{3 \omega}^{*}\right)$ with all elements of $L_{v}$. If Label $\left(\omega, l_{1 \omega}^{*}, l_{2 \omega}^{*}, l_{3 \omega}^{*}\right)$ is the Pareto solution, $\left(\omega, l_{1 \omega}^{*}, l_{2 \omega}^{*}, l_{3 \omega}^{*}\right)$ is memorized to a set of $L_{v}$.

STEP 4-5: Go to STEP 4-6 if all $\left(\sigma, l_{1 \sigma}, l_{2 \sigma}, l_{3 \sigma}\right)$ are selected in STEP 4-2. Go to STEP 4-2 otherwise. STEP 4-6: Go to STEP 5 if all nodes in $W_{v}$ are selected in STEP 4-1. Go to STEP 4-1 otherwise.

STEP 5: (Select the next node or output the Pareto solutions)

STEP 5-1: Go to STEP 5-2 if $v=t$ and if all nodes $v(\in V)$ are selected in STEP 2. Go to STEP 2 after selecting $v^{\prime}\left(\in W_{v}\right)$ and setting $v \leftarrow v^{\prime}$ otherwise.

STEP 5-2: Output all Pareto solutions

$\left\{\left(\tau, l_{1 \tau}, l_{2 \tau}, l_{3 \tau}\right) \mid\left(\left(\tau, l_{1 \tau}, l_{2 \tau}, l_{3 \tau}\right) \in L_{v}\right) \cap(\tau=t)\right\}$, and the algorithm finishes.

Next, we show the algorithm to obtain the Pareto solutions, which is used in STEP 3-4 and STEP 4-4 in the aforementioned algorithm.
Searching procedure for Pareto solutions

STEP 1: Receive $L_{v}$ and $\left(\omega, l_{1 \omega}^{*}, l_{2 \omega}^{*}, l_{3 \omega}^{*}\right)$.

STEP 2: Select $\left(\omega, l_{1 \omega}^{\prime}, l_{2 \omega}^{\prime}, l_{3 \omega}^{\prime}\right)$

$$
\in\left\{\left(\tau, l_{1 \tau}, l_{2 \tau}, l_{3 \tau}\right) \mid\left(\left(\tau, l_{1 \tau}, l_{2 \tau}, l_{3 \tau}\right) \in L_{v}\right) \cap(\tau=\omega)\right\} .
$$

STEP 3: Go to STEP 5 if all of the inequalities $l_{1 \omega}^{*} \geq l_{1 \omega}^{\prime}$, $l_{2 \omega}^{*} \geq l_{2 \omega}^{\prime}$ and $l_{3 \omega}^{*} \geq l_{3 \omega}^{\prime}$ are satisfied. $L_{v} \leftarrow L_{v} \cup$ $\left\{\left(\omega, l_{1 \omega}^{*}, l_{2 \omega}^{*}, l_{3 \omega}^{*}\right)\right\}$, and go to STEP 4 otherwise.

STEP 4: $L_{v} \leftarrow L_{v} \backslash\left\{\left(\omega, l_{1 \omega}^{\prime}, l_{2 \omega}^{\prime}, l_{3 \omega}^{\prime}\right)\right\}$ if all of the inequalities $l_{1 \omega}^{*} \leq l_{1 \omega}^{\prime}, l_{2 \omega}^{*} \leq l_{2 \omega}^{\prime}$, and $l_{3 \omega}^{*} \leq l_{3 \omega}^{\prime}$ are satisfied.

STEP 5: Return $L_{v}$ if all $\left(\omega, l_{1 \omega}^{\prime}, l_{2 \omega}^{\prime}, l_{3 \omega}^{\prime}\right)$ are selected in

STEP 2. Go to STEP 2 otherwise.

\section{Our Algorithms}

In the three-objective network, the extended Dijkstra's algorithm[10] uses a large memory area and takes much computing time to obtain the Pareto solutions. Akiba et al.[10] improved the extended Dijkstra's algorithm so as to reduce the search space. However, this improved algorithm by Akiba et al. cannot obtain all Pareto solutions. So, an efficient and exact algorithm that can obtain all Pareto solutions is required.

We propose a new condition for reducing the search space based on the idea of Takahashi et al.[9]. First, we consider the following one-objective optimal problem with a constraint condition.

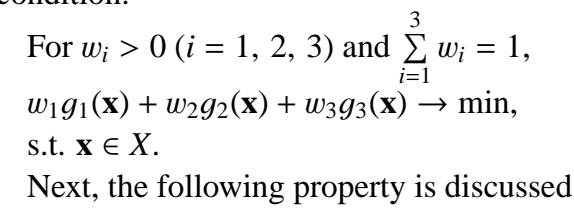

\section{Property 1:}

Let $\mathbf{x}_{s}$ be a path that minimizes $w_{1} g_{1}(\mathbf{x})+w_{2} g_{2}(\mathbf{x})+w_{3} g_{3}(\mathbf{x})$ for $\mathbf{x} \in X$. That is, $\mathbf{x}_{s} \equiv \arg \min _{\mathbf{x} \in X}\left\{w_{1} g_{1}(\mathbf{x})+w_{2} g_{2}(\mathbf{x})+w_{3} g_{3}(\mathbf{x})\right\}$. Then, path $\mathbf{x}_{s}$ is the Pareto solution.

(Proof) If path $\mathbf{x}_{s}$ is not the Pareto solution, at least one path $\mathbf{x}^{\prime}$ dominates $\mathbf{x}_{s}$. From the definition of the Pareto solutions, suppose $g_{1}\left(\mathbf{x}^{\prime}\right)<g_{1}\left(\mathbf{x}_{s}\right), g_{2}\left(\mathbf{x}^{\prime}\right) \leq g_{2}\left(\mathbf{x}_{s}\right)$, and $g_{3}\left(\mathbf{x}^{\prime}\right) \leq g_{3}\left(\mathbf{x}_{s}\right)$ without a loss of generality. Then, $w_{1} g_{1}\left(\mathbf{x}_{s}\right)+w_{2} g_{2}\left(\mathbf{x}_{s}\right)+$ $w_{3} g_{3}\left(\mathbf{x}_{s}\right)>w_{1} g_{1}\left(\mathbf{x}^{\prime}\right)+w_{2} g_{2}\left(\mathbf{x}^{\prime}\right)+w_{3} g_{3}\left(\mathbf{x}^{\prime}\right)$. This is contradictory to the definition of $\mathbf{x}_{s}$. Therefore, Property 1 is proved true.

Figure 2 shows an image of solution (path) $\mathbf{x}_{s}$ in Property 1. Before describing this, each point plotting Figure 2 is explained. As described in section 3, when the edges are gone through, the extended Dijkstra's algorithm obtains the values of objective functions and memorizes them as labels. Such a process can be plotted as the following movement in 3-dimensional space with $g_{1}, g_{2}$, and $g_{3}$ as axes, where $g_{i}$ corresponds to $g_{i}(\mathbf{x})$ for $i=1,2,3$, respectively. Because the start node $s$ corresponds to the origin point in 3-dimensional space, the path starts at the origin point and extends to the direction in which all $g_{i}$ increase, as shown in Figure 3. Therefore, a path to terminal node $t$ can be expressed as a point in 3-dimensional space. In Figure 2, please note that each point corresponds to a path $\mathbf{x}$ from start node $s$ to terminal node $t$, where the coordinate of the point is $\left(g_{1}(\mathbf{x}), g_{2}(\mathbf{x}), g_{3}(\mathbf{x})\right)$. 


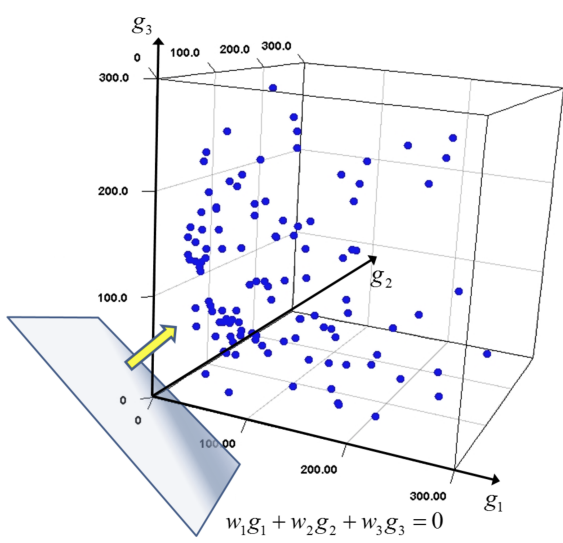

Fig. 2 Image of the path $\mathbf{x}_{s}$ in Property 1

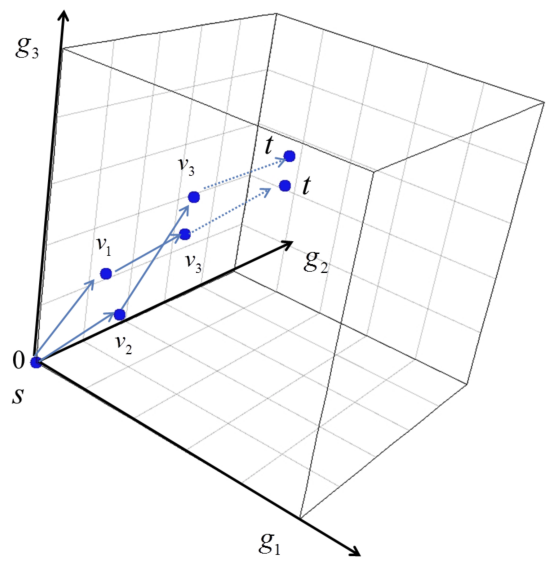

Fig. 3 Image of the process by extended Dijkstra's algorithm

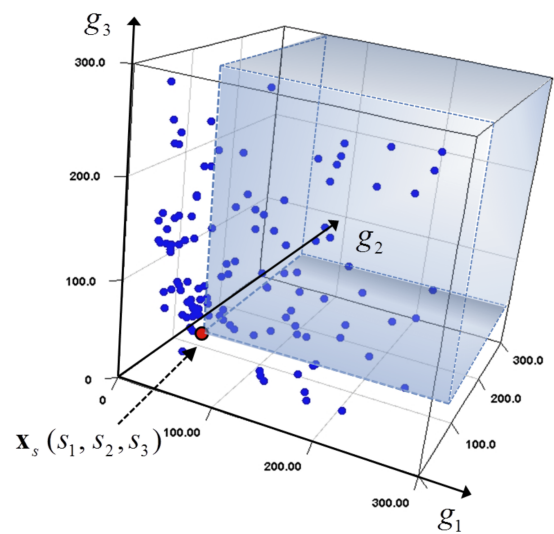

Fig. 4 Reduced search space by path $\mathbf{x}_{s}$

Now, the image of path $\mathbf{x}_{s}$ in Property 1 is described. First, we consider the plane $w_{1} g_{1}+w_{2} g_{2}+w_{3} g_{3}=0$ in 3-dimensional space with $g_{1}, g_{2}$, and $g_{3}$ as axes. We also consider a parallel translation of the plane $w_{1} g_{1}+w_{2} g_{2}+w_{3} g_{3}=0$ to the upper direction. Then, the path $\mathbf{x}_{s}$ stated in Property 1 is expressed as an intersection of the Pareto solutions and the translated plane. For a detailed description, we define notations $s_{1} \equiv g_{1}\left(\mathbf{x}_{\mathbf{s}}\right), s_{2} \equiv$ $g_{2}\left(\mathbf{x}_{\mathbf{s}}\right)$ and $s_{3} \equiv g_{3}\left(\mathbf{x}_{\mathbf{s}}\right)$. Then, path $\mathbf{x}_{s}(\in X)$ is expressed by $\left(s_{1}, s_{2}, s_{3}\right)$. Next, we consider the following property for the Pareto solutions.

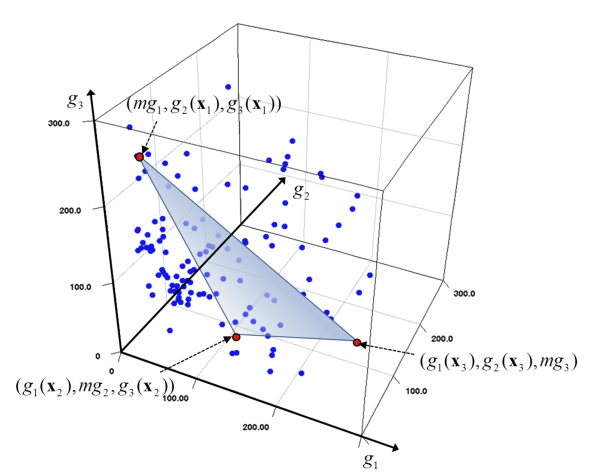

Fig. 5 Plane to reduce search space

\section{Property 2:}

There is no Pareto solution $\mathbf{x}_{p}$ in the space that satisfies all inequalities $g_{1} \geq s_{1}, g_{2} \geq s_{2}$ and $g_{3} \geq s_{3}$.

Property 2 is derived from the definition of the Pareto solutions. Figure 4 illustrates the reduced search space that satisfies the inequalities in Property 2. From this property, our algorithms add the following steps that reduce the search space for the Pareto solutions.

(Add STEP 0 to the extended Dijkstra's algorithm) STEP 0: (Obtain the optimal path for the $\mathbf{x}_{s}$ )

STEP 0-1: Obtain $\mathbf{x}_{s}$ that minimizes $w_{1} g_{1}(\mathbf{x})+w_{2} g_{2}(\mathbf{x})+w_{3} g_{3}(\mathbf{x})$ by using the Dijkstra's algorithm.

STEP 0-2: Obtain vector $\left(s_{1}, s_{2}, s_{3}\right)$ by the path $\mathbf{x}_{s}=\arg \min _{\mathbf{x} \in X}\left\{w_{1} g_{1}(\mathbf{x})+w_{2} g_{2}(\mathbf{x})+w_{3} g_{3}(\mathbf{x})\right\}$ from STEP 0-1.

(Substitute the following STEPs for STEP 3-3 and STEP 4-3 of the extended Dijkstra's algorithm)

STEP 3-3: Calculate $\left(\omega, l_{1 v}+c_{1 e}, l_{2 v}+c_{2 e}, l_{3 v}+c_{3 e}\right)$ for edge $e$ when start $(e)$ is $v$ and $e n d(e)$ is $\omega$, and set $\left(\omega, l_{1 \omega}^{*}, l_{2 \omega}^{*}, l_{3 \omega}^{*}\right) \leftarrow\left(\omega, l_{1 v}+c_{1 e}, l_{2 v}+c_{2 e}, l_{3 v}+c_{3 e}\right)$. Go to STEP 3-4 if one of the following conditions is satisfied:

(a) $l_{1 \omega}^{*}<s_{1}$,

(b) $l_{2 \omega}^{*}<s_{2}$,

(c) $l_{3 \omega}^{*}<s_{3}$.

Go to STEP 3-5 otherwise.

STEP 4-3: Calculate $\left(\omega, l_{1 \sigma}+c_{1 e}, l_{2 \sigma}+c_{2 e}, l_{3 \sigma}+c_{3 e}\right)$ for edge e when $\operatorname{start}(e)$ is $\sigma$ and end $(e)$ is $\omega$, and set $\left(\omega, l_{1 \omega}^{*}, l_{2 \omega}^{*}, l_{3 \omega}^{*}\right) \leftarrow\left(\omega, l_{1 \sigma}+c_{1 e}, l_{2 \sigma}+c_{2 e}, l_{3 \sigma}+c_{3 e}\right)$. Go to STEP 4-4 if one of the following conditions is satisfied:
(a) $l_{1 \omega}^{*}<s_{1}$,
(b) $l_{2 \omega}^{*}<s_{2}$
(c) $l_{3 \omega}^{*}<s_{3}$.
Go to STEP 4-5 otherwise.

\section{Numerical Experiment}

Our algorithms can set bounds to search within less space than the extended Dijkstra's algorithm[10]. However, our algorithms add the procedure to obtain the path $\mathbf{x}_{s}$ that minimizes $w_{1} g_{1}(\mathbf{x})+w_{2} g_{2}(\mathbf{x})+w_{3} g_{3}(\mathbf{x})$. Thus, we compare our algorithms 
Table 1 Comparison of comuting time (sec.)

\begin{tabular}{|c|c|c|c|c|c|c|c|c|c|}
\hline \multirow{3}{*}{ Pattern } & \multirow{2}{*}{ Num. of nodes } & \multirow{2}{*}{ Extended Dikstra } & \multicolumn{3}{c|}{$\begin{array}{c}\text { Proposed Algorithm 1 } \\
\left(w_{1}=w_{2}=w_{3}=1 / 3\right)\end{array}$} & \multicolumn{3}{|c|}{$\begin{array}{c}\text { Proposed Algorithm } 2 \\
\left(w_{i} \text { is coefficient of plane function }\right)\end{array}$} \\
\cline { 2 - 11 } & & Time & SD & Time & Proportion & SD & Time & Proportion & SD \\
\hline \multirow{2}{*}{1} & 100 & 0.274 & 0.063 & 0.034 & $12.60 \%$ & 0.011 & 0.084 & $30.80 \%$ & 0.059 \\
\cline { 2 - 11 } & 1000 & 221.594 & 22.364 & 57.215 & $25.80 \%$ & 13.391 & 113.708 & $51.30 \%$ & 48.363 \\
\hline \multirow{2}{*}{2} & 100 & 0.295 & 0.045 & 0.115 & $39.10 \%$ & 0.073 & 0.159 & $54.00 \%$ & 0.123 \\
\cline { 2 - 11 } & 1000 & 483.38 & 43.687 & 269.228 & $55.70 \%$ & 116.476 & 360.688 & $74.60 \%$ & 137.931 \\
\hline
\end{tabular}

Table 2 Comparison of the number of labels

\begin{tabular}{|c|c|c|c|c|c|c|c|c|c|}
\hline \multirow{2}{*}{ Pattern } & \multirow{2}{*}{ Num. of nodes } & \multicolumn{2}{|c|}{ Extended Djikstra } & \multicolumn{3}{|c|}{$\begin{array}{l}\text { Proposed Algorithm 1 } \\
\left(w_{1}=w_{2}=w_{3}=1 / 3\right)\end{array}$} & \multicolumn{3}{|c|}{$\begin{array}{c}\text { Proposed Algorithm } 2 \\
\left(w_{i} \text { is coefficient of plane function) }\right.\end{array}$} \\
\hline & & Num. of labels & SD & Num. of labels & Proportion & SD & Num. of labels & Proportion & SD \\
\hline \multirow[t]{2}{*}{1} & 100 & 490,740 & 60,514 & 45,978 & $9.4 \%$ & 11,298 & 128,422 & $26.2 \%$ & 104,362 \\
\hline & 1000 & $119,267,200$ & $5,982,838$ & $23,732,852$ & $19.9 \%$ & $5,598,035$ & $56,568,995$ & $47.4 \%$ & $27,947,195$ \\
\hline \multirow[t]{2}{*}{2} & 100 & 534,220 & 38,292 & 199,506 & $37.3 \%$ & 152,022 & 242,231 & $45.3 \%$ & 196,254 \\
\hline & 1000 & $193,898,200$ & $10,339,268$ & $108,868,133$ & $56.1 \%$ & $47,815,928$ & $144,054,941$ & $74.3 \%$ & $62,952,942$ \\
\hline
\end{tabular}

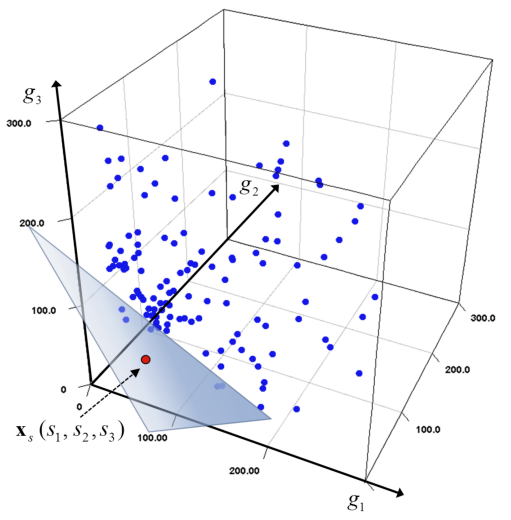

Fig. 6 Image of the path $\mathbf{x}_{s}$ in Algorithm 2

with the extended Dijkstra's algorithm in terms of computing time and the number of labels. The number of labels influences the needed memory area for obtaining the Pareto solutions. Numerical experiments were conducted by using a $\mathrm{PC}$ with an Intel Core i7 CPU (2.4 GHz), 16.0 GB of memory, and MSWindows 8.1 Update 64 bit. The used program was written in C programming language and compiled by Visual Studio 2013. We conducted the experiment using networks where the numbers of nodes were between 10 and 1000. For $i=1,2, \cdots, n$, cost vector $\left(c_{1 i}, c_{2 i}, c_{3 i}\right)$ was prepared using the following two patterns.

\section{Pattern 1:}

$\left(c_{1 i}, c_{2 i}, c_{3 i}\right)$ was generated using uniform random values from 1 to 100 .

\section{Pattern 2:}

$c_{1 i}, c_{2 i}$, and $c_{3 i}$ were generated using uniform random values from 1 to 100,1 to 1000 , and 1 random to 10000 , respectively.

We considered the following two algorithms with respect to $w_{1}, w_{2}$, and $w_{3}$, which influence the search for $\mathbf{x}_{s}$.

Algorithm 1:

$$
w_{1}=w_{2}=w_{3}=\frac{1}{3}
$$

\section{Algorithm 2:}

Define $X_{i} \equiv\left\{\mathbf{x} \mid g_{i}(\mathbf{x})=m g_{i}\right\}$, where $m g_{i} \equiv$ $\min _{\mathbf{x} \in X}\left\{g_{i}(\mathbf{x})\right\}$. For $\mathbf{x}_{i} \in X_{i}$, obtain three vectors $\left(m g_{1}, g_{2}\left(\mathbf{x}_{1}\right), g_{3}\left(\mathbf{x}_{1}\right)\right),\left(g_{1}\left(\mathbf{x}_{2}\right), m g_{2}, g_{3}\left(\mathbf{x}_{2}\right)\right)$, and $\left(g_{1}\left(\mathbf{x}_{3}\right), g_{2}\left(\mathbf{x}_{3}\right), m g_{3}\right) . w_{1}, w_{2}$, and $w_{3}$ are coefficients of the plane equation that satisfies these three points.

Figure 5 shows the plane that is generated using the three vectors in Algorithm 2. The optimal path $\mathbf{x}_{s}$ by Algorithm 2 is illustrated in Figure 6.

Each experiment was conducted five times for different networks in cost vectors. Table 1 shows a comparison of the computing time, and Table 2 shows a comparison of the number of labels in cases where the number of nodes is 100 and 1000 . The computing time and the number of labels are the average values of five time experiments. In Table 1 and Table 2, SD stands for standard deviation, and Proportion is the ratio of the proposed algorithms to the extended Djikstra's algorithm in terms of the computing time or the number of labels. We obtained a standard deviation because the generated cost vectors were different in each experiment. If the experiments were conducted several times for the networks with the same cost vectors, our algorithms output the same results. In Table 1 and Table 2, both proposed algorithms reduced the computing time and the number of labels more than the extended Djikstra's algorithm.

Next, we evaluated our algorithms with respect to the number of nodes. Figure 7 and Figure 8 show the comparisons for computing times and the number of labels, respectively, when the number of nodes was varied from 100 to 1000 . For the computing time, proposed algorithm 1 reduced the computing time to $10-30 \%$ in Pattern 1 and to $30-70 \%$ in Pattern 2, compared with the computing time of the extended Djikstra's algorithm. Similarly, proposed algorithm 2 reduced the computing time to 40-60\% in Pattern 1 and to 50-80\% in Pattern 2. For the number of labels, trends were similar to those of the computing time in Pattern 1 and Pattern 2. Of the two proposed algorithms, Algorithm 1 was more efficient, but its efficiency seemed to change depending on the condition of the cost vectors.

\section{Conclusions}

In this study, we considered the three-objective network design problem. Our study focused on the effective point in reduc- 


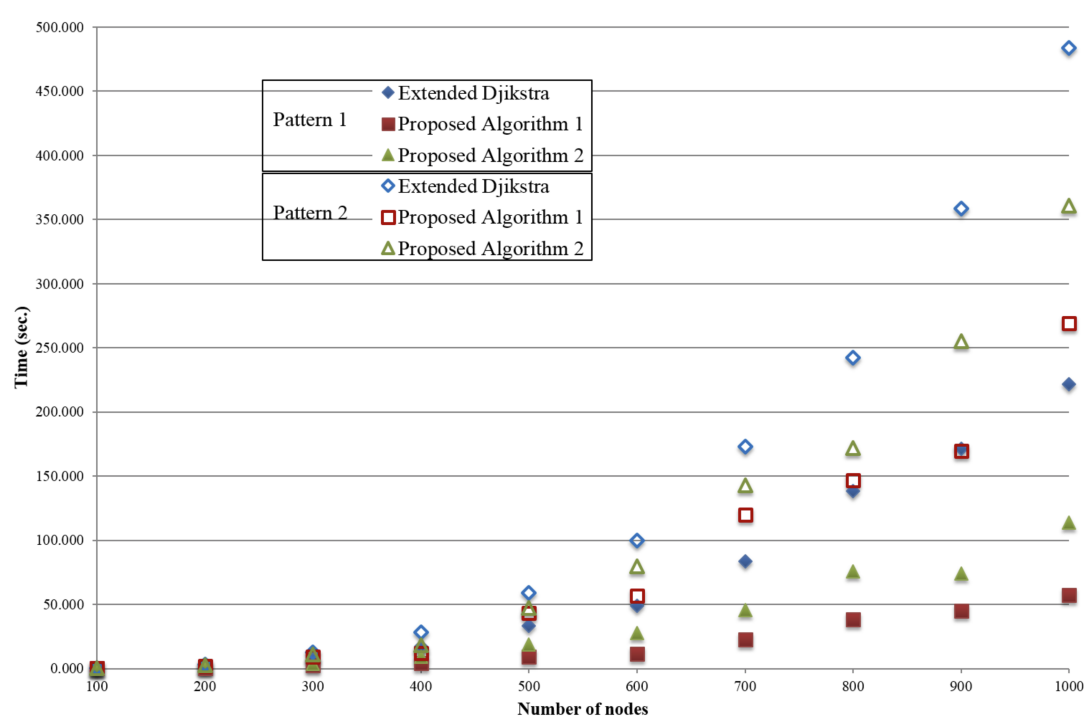

Fig. 7 Comparison of computing time in Pattern 1 and Pattern 2

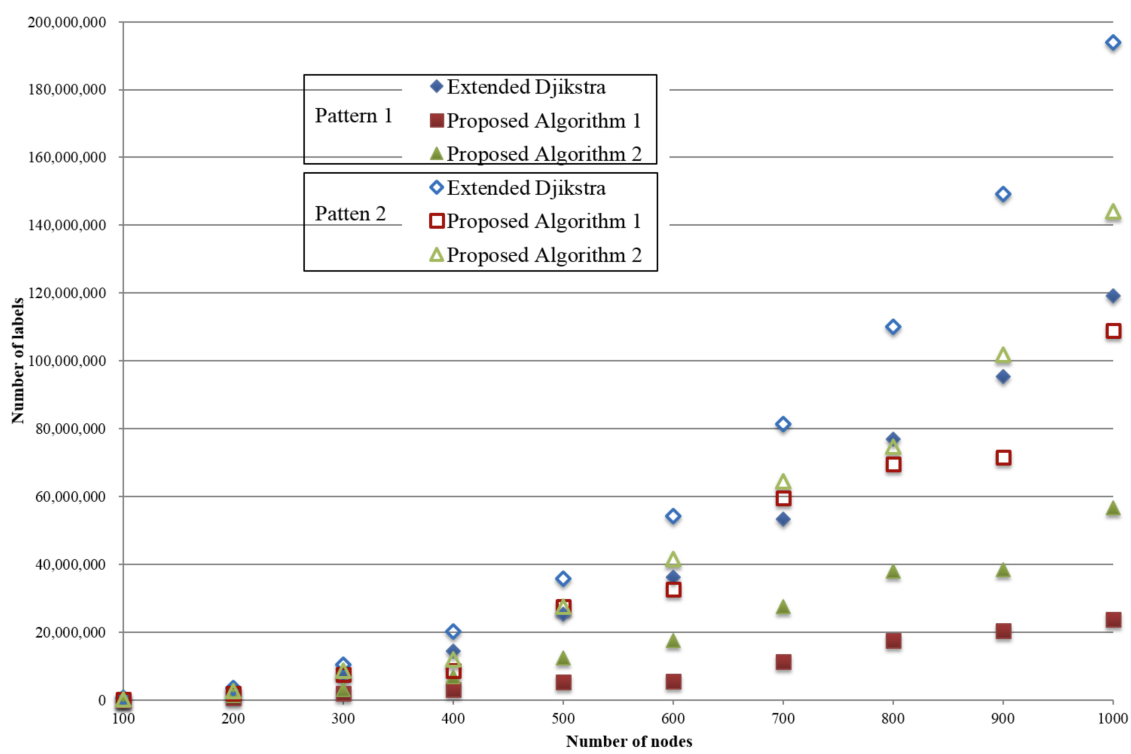

Fig. 8 Comparison of number of labels in Pattern 1 and Pattern 2

ing search space. Using this point, we proposed efficient algorithms for obtaining Pareto solutions. Numerical experiments were conducted to compare our algorithms with the extended Djikstra's algorithm[10] in terms of computing time and number of labels. As the results of our comparisons, the numerical experiments suggested that our algorithms took less computing time and less memory area than the extended Djikstra's algorithm. Our algorithms can be extended to search an n-objective network for Pareto solutions.

Both proposed algorithms reduced the search space, and both were efficient. However, we found that the efficiency varied depending on the means of obtaining the path $\mathbf{x}_{s}$. We will apply a different path as an essential point to reduce the search space as part of our future work.

\section{Acknowledgments}

This work was partially supported by Grant $\sharp 25350456$, a Grant-in-Aid for Scientific Research (c) from JSPS(2013-).
The authors thank the JSPS for their support.

\section{References}

[1] Dijkstera E W, 1959. A note on two problems in connection with graphs, Numerische Mathematik, 1, pp.269-271.

[2] Bellman R, 1958. On a Routing Problem, Quarterly of Applied Mathematics, 16(1), pp.87-90.

[3] Ford L R, Fulkerson D R, 1962. FLOWS in Networks, Princeton University Press.

[4] Hara K, Tsukahara S, Kanoh H, Sota T, Kurokawa H, 2006. Route Planning for Car Navigation Systems Using MultiObjective Genetic Algorithm and Predicted Traffic, Proc. Information Processing Society of Japan 2006, pp.31-38 (in Japanese)

[5] Kambayashi Y, Yamachi H, Tsujimura Y, Yamamoto H, 2007, . Integrating Uncomfortable Intersection-Turns to Subjectively Optimal Route Selection Using Genetic Algorithm, Proc. 5th IEEE Int. Conf. on Computational Cybernetics, pp.203-208.

[6] Aneja Y P, Aggarwal V, Nair K P K, 1983, . Shortest Chain Subject to Side Constrains, Networks, 13, pp.295-302. 
[7] Miyamoto Y, 2002. Experimental of Analysis for Constrained Shortest Path Problem, Proc. Information Processing Society of Japan 2002, pp.35-42 (in Japanese).

[8] Akiba T, Yamamoto H, Egashira T, Iwakami S, 2012. Fast Algorithm for the Pareto Solutions of a Two-Objective Network, Journal of the Society of Plant Engineers Japan, 24(2), pp.4959 (in Japanese).

[9] Takahashi N, Akiba T, Nomura S, Yamamoto H, 2015. An Approach for the Fast Calculation Method of Pareto Solutions of a Two-objective Network, International Journal of Reliability, Quality and Safety Engineering, 22(1), pp.15500051155000513.

[10] Akiba T, Yamamoto H, Rui D Q, Nagatsuka H, 2012. A Fast Calculation Method for the Partial Group of All Pareto Solutions at a Three-objective Network, International Journal of Quality Technology and Quantitative Management, 9(3), pp.305-316.

\section{Natsumi TAKAHASHI}

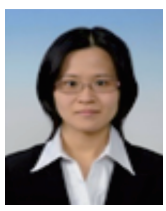

is a doctoral student in Faculty of System Design, Tokyo Metropolitan University. She received the B.S. in Economics from Meiji Gakuin University in 2011, and the M.S. in System Design from Tokyo Metropolitan University, Japan in 2013. Her research interests are optimizations based on the reliability engineering and operations research for network system.

\section{Hisashi Yамамото (Member)}

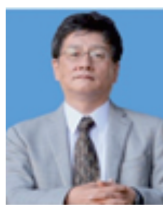

is a Professor in Faculty of System Design, Tokyo Metropolitan University. He received the B.S., M.S. and Doctoral degree (Dr. Eng.) in industrial engineering from Tokyo Institute of Technology, Japan. His main research interests are optimizations based on the reliability engineering. He worked as the Chair of IEEE Reliability Society Japan Chapter, the Editor-in-chief of JIMA and REAJ. He was the Chair of APARM2012 program committee, General Co-chair of APARM2014 and a member of IEICE EA committee.

\section{Tomoaki AKIBA}

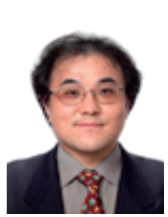

is an Professor in Faculty of Social Systems Science, Chiba Institute of Technology. He received B.S. and M.S. from the Teikyo University of Science and Technology in 1995 and 1997, respectively. He received the doctor' s degree (Dr. Eng.) from Tokyo Metropolitan Institute of Technology in 2004. From 1997 to 2012, he had served as a Lecturer in the Information Management Engineering course of Yamagata College of Industry and Technology. His research interests are optimization based on the reliability modeling, operations research, and so on.

\section{Xiao XIAO}

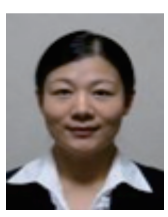

is an Assistant Professor in Faculty of System Design, Tokyo Metropolitan University. She received the B.Eng., M.Eng., and Ph.D.(Engineering) from Hiroshima University, Japan, in 2008, 2010, and 2013, respectively. She worked for six months as an Assistant Professor in Graduate School of Engineering, Hiroshima University. Her research areas include software reliability engineering, performance evaluation, time series analysis, and network design. She is a regular member of ACM, IEEE, IEICE, IPSJ, JIMA, and ORSJ. 